\title{
Surface waves on multilayer hyperbolic metamaterials: Operator approach to effective medium approximation
}

Popov, Vladislav; Lavrinenko, Andrei V.; Novitsky, Andrey

Published in:

Physical Review B

Link to article, DOI:

10.1103/PhysRevB.97.125428

Publication date:

2018

Document Version

Publisher's PDF, also known as Version of record

Link back to DTU Orbit

Citation (APA):

Popov, V., Lavrinenko, A. V., \& Novitsky, A. (2018). Surface waves on multilayer hyperbolic metamaterials: Operator approach to effective medium approximation. Physical Review B, 97(12), [125428].

https://doi.org/10.1103/PhysRevB.97.125428

\section{General rights}

Copyright and moral rights for the publications made accessible in the public portal are retained by the authors and/or other copyright owners and it is a condition of accessing publications that users recognise and abide by the legal requirements associated with these rights.

- Users may download and print one copy of any publication from the public portal for the purpose of private study or research.

- You may not further distribute the material or use it for any profit-making activity or commercial gain

- You may freely distribute the URL identifying the publication in the public portal 


\title{
Surface waves on multilayer hyperbolic metamaterials: Operator approach to effective medium approximation
}

\author{
Vladislav Popov* \\ Department of Theoretical Physics and Astrophysics, Belarusian State University, Nezavisimosti avenue 4, 220030 Minsk, Republic of Belarus \\ and SONDRA, CentraleSupélec, Université Paris-Saclay, F-91190 Gif-sur-Yvette, France \\ Andrei V. Lavrinenko ${ }^{\dagger}$ \\ DTU Fotonik, Technical University of Denmark, Ørsteds Plads 343, DK-2800 Kongens Lyngby, Denmark \\ Andrey Novitsky \\ Department of Theoretical Physics and Astrophysics, Belarusian State University, Nezavisimosti avenue 4, 220030 Minsk, Republic of \\ Belarus and DTU Fotonik, Technical University of Denmark, Ørsteds Plads 343, DK-2800 Kongens Lyngby, Denmark
}

(Received 17 August 2017; revised manuscript received 7 March 2018; published 23 March 2018)

\begin{abstract}
In this paper, we elaborate on the operator effective medium approximation developed recently in Popov et al. [Phys. Rev. B 94, 085428 (2016)] to get insight into the surface polariton excitation at the interface of a multilayer hyperbolic metamaterial (HMM). In particular, we find that HMMs with bilayer unit cells support the TE- and TM-polarized surface waves beyond the Maxwell Garnett approximation due to the spatial dispersion interpreted as effective magnetoelectric coupling. The latter is also responsible for the dependence of surface wave propagation on the order of layers in the unit cell. Elimination of the magnetoelectric coupling in three-layer unit cells complying with inversion symmetry restores the qualitative regularity of the Maxwell Garnett approximation, as well as strongly suppresses the influence of the order of layers in the unit cell.
\end{abstract}

DOI: 10.1103/PhysRevB.97.125428

\section{INTRODUCTION}

A surface wave is usually treated as an electromagnetic wave localized near the interface between two different media, with fields' tails fading exponentially away from the interface. Electromagnetic waves coupled to the surface dipole (e.g., plasma) excitations are called surface polaritons (SPs).

Surface polaritons attract a fair amount of interest because of their prospects for applications. Since the electromagnetic field of SPs is confined to a tiny region near the interface (on a nanometer scale at optical frequencies), one can harness SPs to control the fields on a deeply subwavelength scale. This can lead to the beneficial miniaturization of photonic devices and their performance (speed and energy supply) improvement [1]. SPs on the interface of a sample and sharp metallic probe (scanning plasmon near-field microscopy [2]) can be used to overcome the diffraction limit imposed by conventional optics. Surface polaritons have great potential in biological sensing and label-free detection of chemicals [3,4]. They can effectively assist with the control of radiation of quantum emitters placed at the interface [5,6].

The history of surface electromagnetic waves dates back to the Zenneck waves propagating along the plane interface between dielectric and conducting surfaces at radio frequencies and Wood's (or grating) anomalies in optics [7,8]. The

\footnotetext{
*uladzislau.papou@ centralesupelec.fr

†alav@fotonik.dtu.dk

‡anov@fotonik.dtu.dk
}

anomalies were consistently explained using the excitation of surface polaritons on the flat interface between the air and metal. Since in this case the surface electromagnetic waves are coupled to the induced electron surface charges, they are called surface plasmon polaritons (SPPs). In general, SPPs are the eigenstates of the flat interface between semi-infinite dielectric and metallic media. But there are other types of surface polaritons (phonon, exciton, and Dyakonov's polaritons) that depend on the properties of adjacent media.

Unlike the SPPs that exist due to the opposite signs of dielectric functions of the contiguous media [9], the Dyakonov surface waves emerge due to different crystallographic symmetries of media forming the interface [10]. Being excited on the interface between isotropic dielectric and uniaxial dielectric crystals with the optical axis in the plane of the interface, these surface waves are allowed to propagate (exist) only in certain directions.

Artificial periodic photonic structures provide one more way to control properties of surface waves. They are commonly called Tamm's surface electromagnetic waves, with the name borrowed from solid-state physics. Bloch surface waves exist on the interface of the periodic structure when the adjacent medium is a dielectric. The term "Bloch surface wave" can be misleading as the word "Bloch" usually implies periodicity in the direction of propagation. However, this term is widely used by the community with respect to surface waves propagating along the interface of a periodic structure $[3,11,12]$.

One-dimensional multilayer structures are the simplest artificial electromagnetic materials with a well-developed theory [13]. The interaction of multilayers with electromagnetic fields 
has been studied for more than two centuries, eventually resulting in the creation of a number of optical devices, such as antireflecting coatings, dielectric mirrors, polarizing beamsplitters, filters, waveguides, etc. Layered structures once again attracted a great deal of attention shortly after the concept of indefinite and hyperbolic metamaterials (HMMs) was proposed $[14,15]$. By definition, HMMs are the class of anisotropic media possessing hyperboloidal isofrequency surfaces, which can be implemented using alternating isotropic dielectric and metal subwavelength layers. The promising applications of HMMs are in the field of nano-optics: nanolithography [16], subwavelength imaging [17-19], engineering of the Purcell factor [20,21], etc.

The usage of multilayers in applications becomes more visual if it is possible to replace a periodic composite with the equivalent homogeneous medium, i.e., to homogenize the multilayer. The Maxwell Garnett approximation is ordinarily exploited for homogenization of deeply subwavelength layered structures giving local effective material parameters. Although the Maxwell Garnett approximation is appealing due to its simplicity, it may fail in some special situations. In Refs. [22,23] it was demonstrated that the Maxwell Garnett approach often overestimates the Purcell factor used for engineering the emitters' lifetime. The Maxwell Garnett approximation also contradicts theoretical [24,25] and experimental [26] research on the behavior of periodic all-dielectric mutilayers with deeply subwavelength unit cells near the critical angle of the total internal reflection. The inadequacy of the Maxwell Garnett approximation is explained by the presence of the spatial dispersion in the multilayer [27], which is relevant to light transmission near the critical angle as well [28].

Generalization of homogenization for periodic metamaterials developed in Ref. [29] was used in Ref. [30] with regard to layered HMMs. This approach proposes the exact solution to the problem and describes the system in terms of the effective dielectric permittivity tensor alone. The latter assertion implies that the magnetization currents are eliminated from the constitutive equations [29], and the tangential components of the magnetic field are discontinued across the interface of the effective medium, which makes solving the boundary problem more difficult. However, the exact solution for HMM homogenization obtained in Ref. [30] is too cumbersome to analyze in the closed form. Recently, Ref. [28] gave a different perspective on the generalization of the homogenization exploiting the operator technique. The operator approach to the effective medium theory allows describing the structure in terms of four material tensors: dielectric permittivity, magnetic permeability, and two gyration pseudotensors. The material tensors are written in the form of a series over small $k_{0} d$, where $k_{0}$ is the vacuum wave number of the electromagnetic wave and $d$ is the period of the structure. The material parameters up to $\left(k_{0} d\right)^{2}$ take into account spatial dispersion and can be analyzed analytically.

In this paper we consider surface waves on the interface between the semi-infinite isotropic medium and periodic layered metal-dielectric structure, as shown in Fig. 1. We deal with subwavelength layered structures of two types: (i) one with a bilayer unit cell (metal-dielectric and dielectric-metal) and (ii) one with a three-layer unit cell possessing the center of inversion (metal-dielectric-metal and dielectric-metal-dielectric).

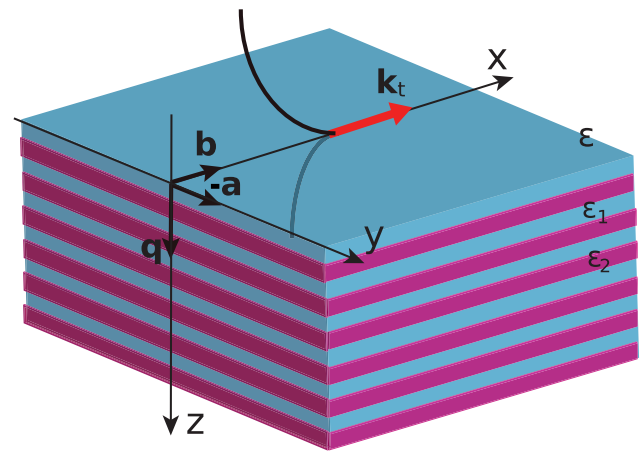

FIG. 1. Sketch of the surface wave propagating on the interface $z=0$ between the semi-infinite dielectric (permittivity $\varepsilon$ ) and multilayer media.

We investigate the influence of spatial dispersion of HMMs on propagation of surface waves in the above-mentioned configurations of the unit cell. Using the operator effective medium approximation we reveal the origin of different branches of dispersion curves for TM- and TE-polarized surface waves and derive the criterion to choose the unit-cell structure which validates the Maxwell Garnett approximation.

This paper is organized as follows. In Sec. II, the structures with the bilayer and three-layer unit cells are homogenized in accordance with the operator approach. Wave impedances of eigenwaves in the effective medium and Bloch waves in the layered medium are discussed in Sec. III. The derivation of dispersion equations and comparison of dispersion curves of TM- and TE-polarized surface waves within the exact theory, Maxwell Garnett approximation, and operator approach are presented in Sec. IV. Section V concludes the paper.

\section{UNIT-CELL-BASED HOMOGENIZATION}

Since subwavelength stratified structures possess strong spatial dispersion [24,27], we aim to reveal its influence on surface electromagnetic states using the operator effective medium approximation (OEMA) developed in Refs. [28,31]. The OEMA allows one to homogenize a planar multilayer structure with an arbitrary unit cell. When the unit-cell thickness $d$ is small in comparison with wavelength $\lambda$, the OEMA provides effective medium parameters in terms of decomposition in $\left(k_{0} d\right)$. Here $k_{0}=2 \pi / \lambda=\omega / c$ is the vacuum wave number, and $\omega$ is the angular frequency. Time dependence $\exp (-i \omega t)$ is assumed throughout the paper. The maximum power of $\left(k_{0} d\right)$ kept in the effective medium parameter decomposition defines the OEMA order.

Effective medium parameters within the Maxwell Garnett approximation do not depend on the position of layers in a unit cell, but the accurate solution indeed does. In this section we show that the structure of the unit cell can be captured by the operator effective medium approximation. We consider bilayer and three-layer unit cells composing multilayer structures. The bilayer unit cell is a couple of isotropic metallic and dielectric slabs with permittivities $\varepsilon_{m}$ and $\varepsilon_{d}$ and thicknesses $d_{m}$ and $d_{d}$, respectively. We distinguish two structures depending on the order of layers, as in the insets of Figs. 2(a) and 2(b). The threelayer unit cell has the inversion center and can be obtained from 

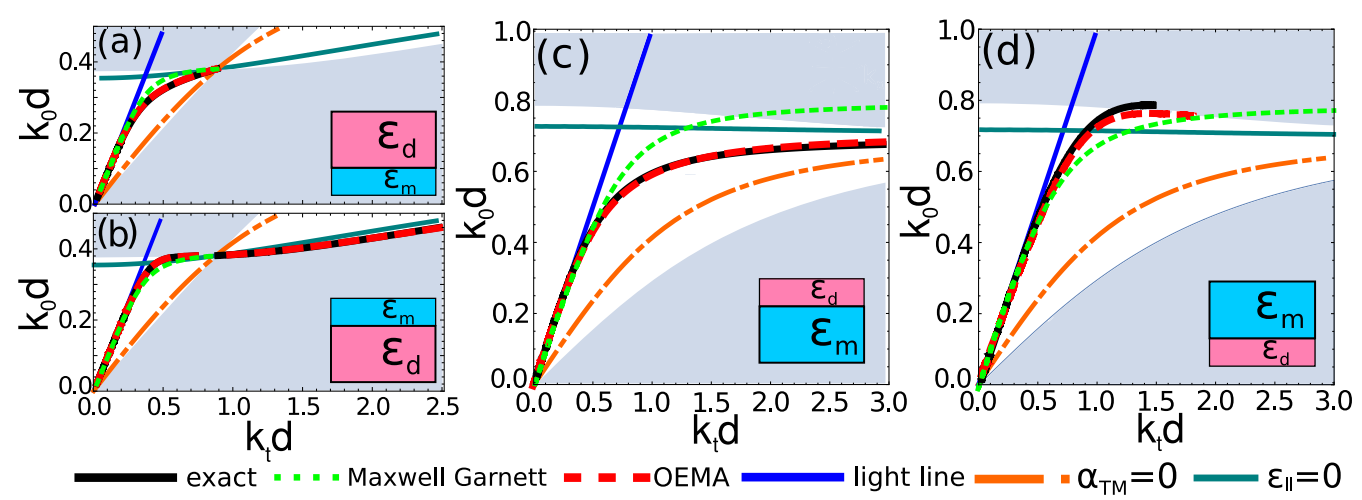

FIG. 2. Dispersion curves of surface polaritons on the semi-infinite multilayer with the bilayer unit cell: (a) metal fill fraction $\rho=0.2$ and upper dielectric layer in the unit cell, (b) $\rho=0.2$ and upper metallic layer, (c) $\rho=0.6$ and upper dielectric layer, and (d) $\rho=0.6$ and upper metallic layer. Effective dielectric permittivity $\varepsilon_{||}$is positive above the curve $\varepsilon_{\|}=0$. The magnetoelectric coupling coefficient is positive above the orange dash-dotted curve $\alpha_{T M}=0$ for (a) and (c) and below the curve for (b) and (d). Shaded areas represent propagation bands within the second-order OEMA. Parameters: $\varepsilon=1, \varepsilon_{d}=4$, and $\omega_{p}=2 \pi c /(4 d)$.

the bilayer unit cell if half of the upper layer is replaced at the bottom, as demonstrated in the insets of Figs. 3(a) and 3(b). Again, two unit cells are possible depending on the order of slabs.

Propagation of electromagnetic waves in the multilayer structure can be described as in Appendix A. On the other hand, the subwavelength layered structure can be replaced with the homogeneous effective medium with permittivity $\hat{\varepsilon}_{\text {eff }}$ and permeability $\hat{\mu}_{\text {eff }}$ tensors and gyration pseudotensors $\hat{\alpha}_{\text {eff }}, \hat{\beta}_{\text {eff }}$ [28]. The zeroth-order OEMA reproduces the Maxwell Garnett approximation for any of the considered unit cells: the effective medium is a nonmagnetic $\left(\hat{\mu}_{\text {eff }}=\hat{1}\right)$ and nongyrotropic $\left(\hat{\alpha}_{\text {eff }}=\right.$ $\left.\hat{\beta}_{\text {eff }}=\hat{0}\right)$ uniaxial crystal, with the dielectric permittivity tensor being equal to

$$
\begin{aligned}
& \hat{\varepsilon}_{\mathrm{eff}}=\varepsilon_{\|}^{(0)} \hat{I}+\varepsilon_{\perp}^{(0)} \mathbf{q} \otimes \mathbf{q}, \\
& \varepsilon_{\|}^{(0)}=\rho \varepsilon_{m}+(1-\rho) \varepsilon_{d}, \quad \frac{1}{\varepsilon_{\perp}^{(0)}}=\frac{\rho}{\varepsilon_{m}}+\frac{1-\rho}{\varepsilon_{d}},
\end{aligned}
$$

where $\mathbf{q}$ is the unit vector orthogonal to the plane of layers (see Fig. 1), $\mathbf{q} \otimes \mathbf{q}$ is the outer (tensor) product of vectors $\mathbf{q}$ defined as $(\mathbf{q} \otimes \mathbf{q})_{i, j}=q_{i} q_{j}, \hat{I}=\hat{1}-\mathbf{q} \otimes \mathbf{q}$ is the projector operator on the plane of layers, $\hat{1}$ is the three-dimensional unit tensor, and $\rho=d_{m} / d$ is the fill fraction of material 1 . In the Maxwell Garnett approximation, structures depicted in the insets of Figs. 2 and 3 are indistinguishable, being described by the same permittivity tensor equation (1). However, as demonstrated both theoretically and experimentally [24,26], there is a nonvanishing difference in the electromagnetic response depending on the geometry of the unit cells even in the deep-subwavelength regime. This difference can be successfully reproduced within the second-order OEMA [28]. Higher-order corrections are too cumbersome for analytical analysis and have minor impact.

Nonlocal effective material parameters $\hat{\varepsilon}_{\text {eff }}, \hat{\mu}_{\text {eff }}, \hat{\alpha}_{\text {eff }}$, and $\hat{\beta}_{\text {eff }}$ [28] provide higher accuracy in comparison with the Maxwell Garnett approach. The second-order OEMA introduces only the second-order corrections to the Maxwell Garnett permittivity and permeability tensors for both the bilayer and three-layer unit cells as

$$
\begin{aligned}
& \varepsilon_{\|}^{(2)}=\varepsilon_{\|}^{(0)}+\delta \varepsilon_{\|}^{(2)}(b, \omega), \quad \varepsilon_{\perp}^{(2)}=\varepsilon_{\perp}^{(0)}+\delta \varepsilon_{\perp}^{(2)}(b, \omega), \\
& \mu_{\|}^{(2)}=1+\delta \mu_{\|}^{(2)}(b, \omega), \quad \mu_{\perp}^{(2)}=1+\delta \mu_{\perp}^{(2)}(b, \omega),
\end{aligned}
$$

where $\delta \varepsilon_{\|, \perp}^{(2)}(b, \omega)$ and $\delta \mu_{\|, \perp}^{(2)}(b, \omega)$ are proportional to $\left(k_{0} d\right)^{2}$. Explicit expressions for the corrections in the cases of two- and three-layer unit cells can be found in Appendix B. Nonlocality of the homogenized material is manifested as a dependence
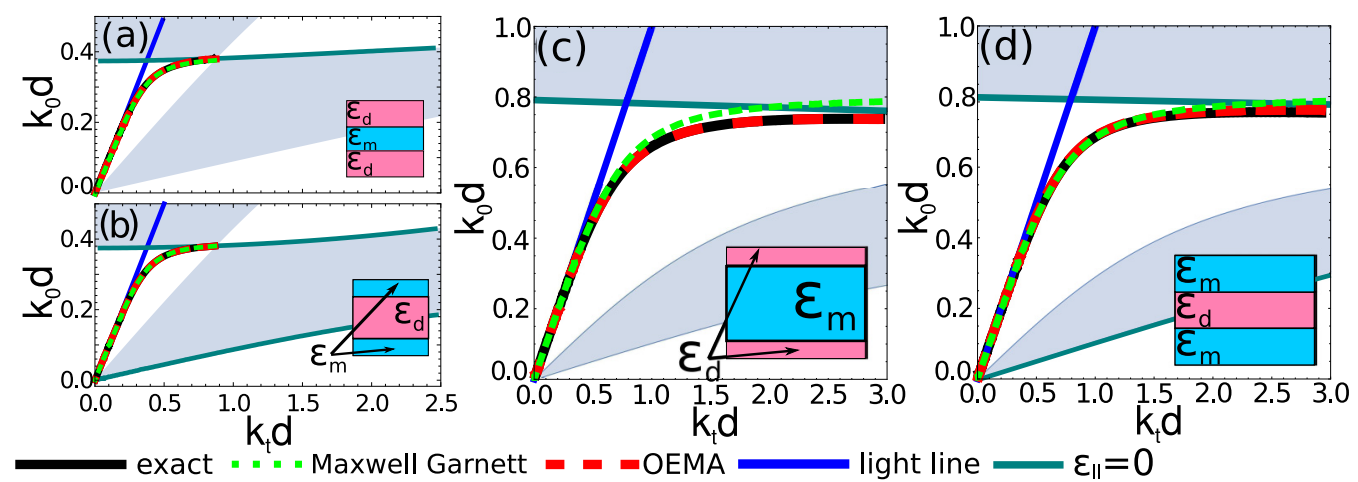

FIG. 3. Dispersion curves of surface polaritons on a semi-infinite multilayer with the three-layer unit cell for different metal fill fractions and orders of layers. The metal fill fraction is (a) and (b) $\rho=0.2$ and (c) and (d) $\rho=0.6$. Effective dielectric permittivity $\varepsilon_{\|}$is positive above the upper curve $\varepsilon_{\|}=0$. Shaded areas represent propagation bands within the second-order OEMA. Parameters: $\varepsilon=1, \varepsilon_{d}=4$, and $\omega_{p}=2 \pi c /(4 d)$. 
of the effective parameters on the normalized tangential wave number $b=k_{t} / k_{0}$. In the context of surface waves, the length of tangential wave vector $\mathbf{k}_{t}=k_{0} \mathbf{b}$ defines the propagation constant of surface waves (see Fig. 1).

Bilayer unit cells are also characterized by the gyration pseudotensors [28]

$$
\hat{\alpha}_{\mathrm{eff}}=-\hat{\beta}_{\mathrm{eff}}^{T}=i \alpha_{\mathrm{TE}} \frac{\mathbf{a} \otimes \mathbf{b}}{b^{2}}+i \alpha_{\mathrm{TM}} \frac{\mathbf{b} \otimes \mathbf{a}}{b^{2}},
$$

where $\mathbf{a}=\mathbf{b} \times \mathbf{q}$ and effective magnetoelectric coupling constants

$$
\begin{gathered}
\alpha_{\mathrm{TE}}=\frac{k_{0} d}{2} \sigma, \quad \alpha_{\mathrm{TM}}=\frac{k_{0} d}{2} \sigma f(b), \\
f(b)=\frac{b^{2}}{\varepsilon_{r}}-1, \quad \varepsilon_{r}=\left(\varepsilon_{m}^{-1}+\varepsilon_{d}^{-1}\right)^{-1} .
\end{gathered}
$$

The parameter $\sigma$ is defined by the sequence of layers, being equal to

$$
\sigma=\rho(1-\rho)\left(\varepsilon_{m}-\varepsilon_{d}\right)<0
$$

when the top layer is the dielectric one [as in the inset of Fig. 2(a)] and

$$
\sigma=\rho(1-\rho)\left(\varepsilon_{d}-\varepsilon_{m}\right)>0
$$

when the metallic layer is the topmost one [see the inset of Fig. 2(b)]. Thus, when one reverses the order of slabs in the stack $(m \leftrightarrow d)$, the parameter $\sigma$ changes its sign and so do $\hat{\alpha}_{\text {eff }}$ and $\hat{\beta}_{\text {eff }}$. There are two mechanisms of spatial dispersion according to Eq. (4), first, the gyrotropy as such and, second, the explicit dependence of $\alpha_{\mathrm{TM}}$ (but not $\alpha_{\mathrm{TE}}$ ) on the tangential wave vector through the function $f(b)$.

Three-layer unit cells have an inversion center that prohibits the existence of the material's gyrotropy $\left(\hat{\alpha}_{\text {eff }}=\hat{\beta}_{\text {eff }}=0\right)$.

In the following section we will study the influence of the unit-cell structure (and therefore magnetoelectric coupling) on the propagation of the surface waves.

\section{ANALYSIS OF SURFACE POLARITON DISPERSION}

Let us now consider surface modes localized at the interface between two semi-infinite media. The first medium is an isotropic dielectric of permittivity $\varepsilon$. The second medium is a multilayer HMM with permittivities of dielectric $\varepsilon_{d}$ and metal $\varepsilon_{m}(\omega)=1-\left(\omega_{p} / \omega\right)^{2}$, where $\omega_{p}$ is the plasma frequency. Electromagnetic waves localized at the interface $z=0$ between semi-infinite adjacent media can be characterized by the wave impedance defined by $\mathbf{q} \times \mathbf{E}_{t}^{m+}=Z_{m}^{+} \mathbf{H}_{t}^{m+}$ for $z>0$ and $\mathbf{q} \times \mathbf{E}_{t}^{m-}=Z_{m}^{-} \mathbf{H}_{t}^{m-}$ for $z<0$. Here $m$ designates TE and TM polarizations, $\mathbf{E}_{t}$ and $\mathbf{H}_{t}$ are the tangential components of electric and magnetic fields, respectively, at the interface $z=0$, and $Z$ is the wave impedance. Applying the conditions of continuity of tangential fields, it can be concluded that the surface mode exists only if the wave impedances of evanescent eigenwaves in both neighboring media coincide, i.e.,

$$
Z_{m}^{-}=Z_{m}^{+}
$$

where $Z^{-}$and $Z^{+}$are the impedances of waves in dielectric $(z<0)$ and multilayer/effective media $(z>0)$, respectively
(Fig. 1). A complete description of the propagation characteristics of the surface waves can be extracted from the dispersion equation (8).

Replacing the HMM with a homogeneous spatially dispersive medium, the dispersion equations for TM- and TEpolarized surface modes take the form (see Appendix $\mathrm{C}$ for details)

$$
\begin{gathered}
\varepsilon_{\|}+\frac{\varepsilon}{\kappa} \alpha_{\mathrm{TM}}=-\frac{\varepsilon}{\kappa} \kappa_{\mathrm{TM}}, \\
\frac{\alpha_{\mathrm{TE}}}{\kappa_{\mathrm{TE}}}=-1-\frac{\mu_{||} \kappa}{\kappa_{\mathrm{TE}}},
\end{gathered}
$$

respectively, where $\kappa>0$ and $\kappa_{T E, T M}>0$ are attenuation constants corresponding to the waves in the dielectric and effective medium regions, respectively. The attenuation constants are defined as $k_{z}=i \kappa k_{0}$ ( $k_{z}$ is the longitudinal wave number). For instance, within the Maxwell Garnett approximation one has to set $\alpha_{\mathrm{TE}}=\alpha_{\mathrm{TM}}=0, \mu_{\|}=\mu_{\perp}=1, \varepsilon_{\|}=\varepsilon_{\|}^{(0)}$, and $\varepsilon_{\perp}=\varepsilon_{\perp}^{(0)}$.

For nonhomogenized periodic multilayers the TE- and TM-polarized surface states follow from Eq. (8), where the impedances of Bloch waves (D4) and (D7) can be found in Appendix D.

\section{A. TM polarization}

We start with the analysis of dispersion equation (9) for TM-polarized surface modes on the interface between the homogenized medium and dielectric $\varepsilon$. This dispersion equation obviously has a solution only if the signs of the left- and right-hand sides coincide. It is important that the peculiar properties of the surface wave dispersion equation related to the spatial dispersion (such as nonzero magnetoelectric coupling $\alpha_{\mathrm{TM}}$ ) are missing in the Maxwell Garnett approximation. $\alpha_{\text {TM }}$ plays a dramatic role in the surface wave phenomena primarily because it is normally much larger than the effective permittivity and permeability corrections. Parameter $\alpha_{\mathrm{TM}} \neq 0$ is realized for bilayer-cell multilayers. In this case the Maxwell Garnett approximation is unable to describe the surface waves in a proper way, as demonstrated in Fig. 2. On the other hand, the gyrotropic response of the three-layer unit cells with the inversion center is absent $\left(\alpha_{\mathrm{TM}}=0\right)$, and therefore, the Maxwell Garnett approach is in good agreement with the exact solution, as seen in Fig. 3. The second-order OEMA almost perfectly describes dispersion of surface polaritons independent of unit-cell structure even when $k_{0} d$ is not much less than unity. Clearly, the breakdown of the Maxwell Garnett approximation originates from the strong spatial dispersion represented here by the magnetoelectric coupling. It is necessary to remember that description of the spatial dispersion is not unique, and similar results could be obtained by means of the nonsymmetric permittivity tensor instead of the magnetoelectric coupling [28].

Let us now discuss the properties of surface wave dispersion curves in detail, paying attention to the order of metallic and dielectric slabs and the dependence on the metal fill fraction $\rho$. For a thick dielectric layer on top of a multilayer [Fig. 2(a)] the effective permittivity is metallic $\left(\varepsilon_{\|}<0\right)$, providing the coupling of electromagnetic waves to plasmons. When the order of the metal and dielectric is reversed [see Fig. 2(b)], the low- $k_{t}$ branch for surface polaritons reaches the propagation band (shaded area), terminates, and recrudesces after the break 


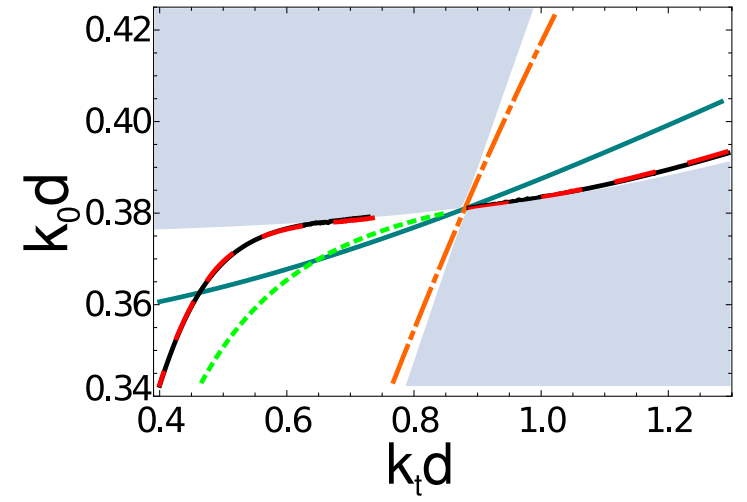

FIG. 4. Close-up of the dispersion diagram in Fig. 2(b) in the vicinity of the break of exact and OEMA dispersion curves.

as a high- $k_{t}$ branch. Notice that for $\varepsilon_{\|}>0$ the existence of surface polaritons can be explained only by the spatial dispersion or, in our interpretation, negative magnetoelectric coupling coefficient $\alpha_{\mathrm{TM}}$ (see Fig. 4).

The high- $k_{t}$ branch squeezes between the allowed propagation band and the curve $\varepsilon_{\|}=0$ and exists owing to the proper sign of $\alpha_{\mathrm{TM}}$ that is not realized in Fig. 2(a).

Now we turn to the influence of the metal fill fraction. In the limiting case $\rho \rightarrow 1$ the surface modes on the multilayer interface change to the surface plasmon polaritons on the metal-dielectric interface. The Maxwell Garnett approach provides a reasonable approximation for the case with an upper dielectric layer [see Figs. 2(a) and 2(c)], revealing behavior similar to that of surface waves on the interface of a uniaxial crystal $[9,32,33]$. The high- $k_{t}$ branch noticed in Fig. 2(b) ceases to exist when $\rho>0.5$ and $\alpha_{\mathrm{TM}}<0$, as in Fig. 2(d).

The significant role of magnetoelectric coupling is more conspicuous for the surface polaritons on the three-layer-cell multilayer shown in the insets of Figs. 3(a) and 3(b). Due to the inversion symmetry of the cell, or, in other words, $\alpha_{\mathrm{TM}}=0$, there is no qualitative change in the surface polariton dispersion depending on the order of layers [see Fig. 3(a) vs Fig. 3(b) and Fig. 3(c) vs Fig. 3(d)]. Moreover, the Maxwell Garnett approximation reproduces the exact dispersion curves quite well, and the second-order OEMA quantitatively improves only the performance of the zeroth order. Thus, to get rid of the noticeable influence of the spatial dispersion on surface polariton dispersion, one needs to exploit the unit cells with the inversion center. Then the interface of the multilayer HMM sustains the surface modes regardless of the order of the layers, and the predictions of the Maxwell Garnett approximation are fulfilled for the subwavelength metal-dielectric multilayers.

In general, the OEMA of the second-order well describes the surface wave dispersion curves that appear in the band gap between the first and second propagation bands in the low-frequency range $k_{0} d<1$. Varying the material parameters has no dramatic effect. Dielectrics of a high refractive index squeeze the band structure, keeping the dispersion curves of surface waves within the considered band gap. On the contrary, extremely low index dielectrics such as epsilon-near-zero (ENZ) materials stretch the band structure. In this case only the band gap right below the first propagation band gets into the low-frequency range $k_{0} d<1$. Still, the second-order OEMA perfectly describes the structure with the three-layer unit cell. However, the case of the bilayer unit cell requires careful analysis taking into account the interplay between $\alpha_{\mathrm{TM}}, \varepsilon_{d}$, and $\varepsilon$.

The strong influence of the order of layers for bilayer unit cells was revealed in Ref. [34]. However, the peculiarities of the structures composed of three-layer unit cells and the ability of the Maxwell Garnett approximation to describe them well have not been presented in the literature to the best of our knowledge.

\section{B. TE polarization}

Although TE-polarized surface modes do not exist on the interfaces of homogeneous crystals when the optical axis is normal to the interface, they can be excited on photonic crystals, whose unit cell size is compared with the operating wavelength (one-dimensional photonic crystals are discussed in Refs. [34-36]). TE-polarized surface waves can also be excited on grounded thin dielectric slabs with large dielectric permittivity [37].

Homogenizing the subwavelength structure within the Maxwell Garnett approximation, we get an effective medium incapable of supporting propagation of TE-polarized surface waves, although the exact solution predicts them. TE-polarized surface waves can be explained in the second-order OEMA governed by Eq. (10). Since $\kappa$ and $\kappa_{\mathrm{TE}}$ are certainly positive, the effective parameters $\alpha_{\mathrm{TE}}$ and $\mu_{\|}$should be responsible for excitation of the TE surface waves. But the effective magnetic response of the multilayers specified by Eqs. (2) is weak, and as demonstrated by Fig. 5, $\mu_{\|}$is always positive. Thus, in spite of the nontrivial magnetic response of the multilayer HMMs, it cannot lead to the existence of TE surface modes on its own. The only reason for the TE-polarized surface waves is the gyrotropic response of multilayers $\alpha_{\mathrm{TE}}<0$. This also means that TE surface waves cannot be supported by the
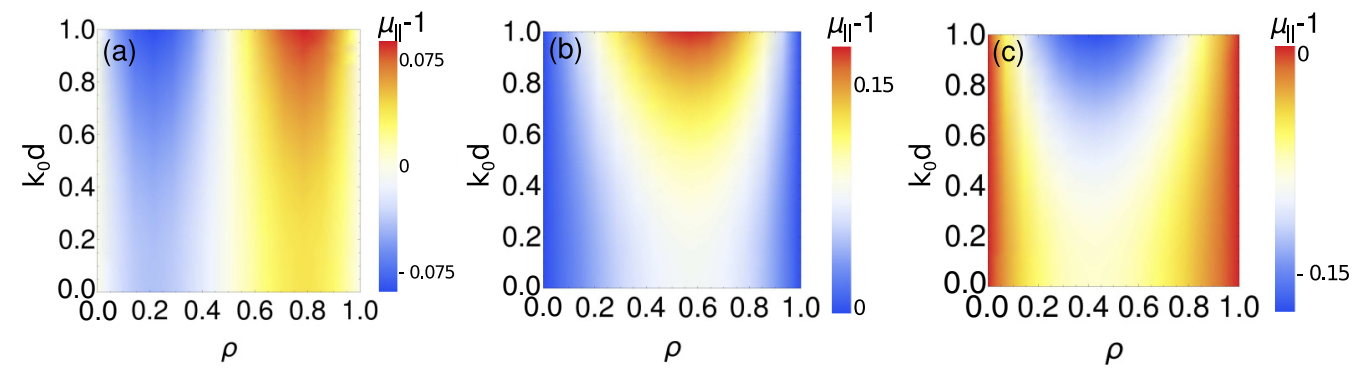

FIG. 5. $\mu_{\|}-1$ vs normalized frequency $k_{0} d$ and metal fill fraction $\rho$ for (a) the bilayer unit cell and the three-layer unit cells with the topmost (b) dielectric and (c) metallic layers. Parameters: $\varepsilon_{d}=4$ and $\omega_{p}=2 \pi c /(4 d)$. 


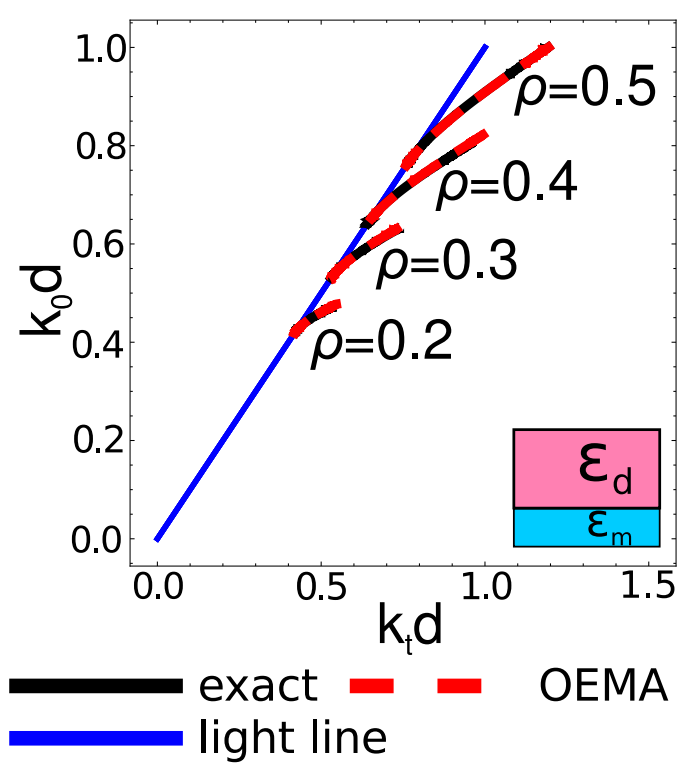

FIG. 6. Dispersion curves of TE-polarized surface modes on the semi-infinite HMM with the bilayer unit cell (topmost dielectric layer) plotted for different metal fill fractions. Parameters: $\varepsilon=1, \varepsilon_{d}=4$, and $\omega_{p}=2 \pi c /(4 d)$.

three-layer-cell HMMs $\left(\alpha_{\mathrm{TE}}=0\right)$, in full accordance with the Maxwell Garnett approximation.

In contrast to the case of TM polarization, the sign of $\alpha_{\mathrm{TE}}$ is determined only by the order of layers in the cell [parameter $\sigma$ in Eq. (4)]. Therefore, only the bilayer-cell structure with the dielectric layer on top of the multilayer supports TE-polarized surface states, while the dispersion curves behave similarly for any metal fill fraction, as shown in Fig. 6. The agreement of the second-order OEMA and exact calculations is almost perfect. Dispersion curves of surface modes terminate when approaching the edge of the first propagation band.

Varying the dielectric permittivity $\varepsilon_{d}$, we keep good agreement between the second-order OEMA and exact dispersion curves within the low-frequency range $k_{0} d<1$. The increase in $\varepsilon_{d}$ does not introduce qualitative changes to the dispersion curves of TE-polarized surface waves. In another extreme case of the ENZ dielectric, the TE surface waves cease to exist because the system effectively behaves like a metal.

Such a counterintuitive existence of TE-polarized surface waves can be explained in terms of OEMA. Since there is only a tangential electric field, the surface charges (as in the case of TM polarization) cannot be induced. However, the magnetoelectric coupling coefficient leads to the existence of the effective tangential magnetization surface current induced in parallel to the electric field, resulting in the discontinuity of the tangential component of the magnetic field across the interface. Thus, TE-polarized surface electromagnetic waves are coupled to the effective surface magnetization currents. Since the currents are not real, TE-polarized surface waves exist without surface excitations.

\section{CONCLUSION}

In this paper, we have employed the operator effective medium approximation to analyze the surface wave propaga- tion on the flat interface between a semi-infinite dielectric and layered hyperbolic metamaterial. We have considered HMM unit cells with (three-layered cell) and without (bilayered cell) an inversion center and studied the influence of the layer order. The surface wave propagation was determined by the dispersion curves, which were obtained within the Maxwell Garnett approximation, second-order OEMA, and exact solution of Maxwell's equations. A direct comparison of the dispersion curves reveals the constraints of the Maxwell Garnett approach and explains the reasons for the peculiarities of the surface wave dispersion.

Within the Maxwell Garnett approximation the order of materials in a unit cell does not matter; only the filling fraction of the constituent materials matters. However, the rigorous calculation manifests a strong dependence of the surface mode propagation on the order of layers if the inversion symmetry is missing (bilayer unit cell). A pronounced demonstration of the effect of the layer ordering comes out when metallic layers are at the edge of the multilayer interface [see Figs. 2(b) and 2(d)]. If $\rho$ is small enough, the structure supports surface polaritons with high tangential wave numbers $k_{t}$. From the OEMA, it follows that such behavior is observed owing to the spatial dispersion (effective magnetoelectric coupling) absent within the Maxwell Garnett approximation. Magnetoelectric coupling is also responsible for the sensitivity of the SP excitation with respect to the order of layers and existence of the TE-polarized surface waves shown in Fig. 6.

Having an inversion center, a three-layer-cell structure has no gyrotropic response. Therefore, the dispersion curves within the Maxwell Garnett approximation resemble those extracted from the accurate calculation, while the second-order OEMA serves only for the quantitative corrections. Thus, the Maxwell Garnett approximation can be reasonably exploited for multilayers with inversion-symmetric unit cells, e.g., threelayer cells.

We emphasize that the predictions of the second-order OEMA agree very well with the exact calculations for both transmission and reflection and surface wave propagation. Transmission and reflection require the equality of evolution operators $\exp \left(i k_{0} z \hat{M}\right)$ of a unit cell and the corresponding effective medium slab (see Appendix B), as discussed in detail in Ref. [28]. The surface wave dispersion equations can be written as the equality of wave impedances (8), where the impedances for the multilayer and effective medium are expressed by means of matrices $\hat{M}$. The surface impedances for semi-infinite homogenized media take into account only the waves leaving the interface (Appendix C). That is why they are simpler than the impedances of periodic structures capturing multiple reflections of plane waves from the interfaces within the cell (Appendix D). Thus, the OEMA comprehensively explains the origin of the TM and TE surface polariton peculiarities on the HMM interface by means of the effective magnetoelectric coupling. Our results could be useful for various applications, including sensors and near-field microscopy.

\section{ACKNOWLEDGMENTS}

V.P. and A.N. thank the Belarusian Republican Foundation for Fundamental Research (Grant No. F16R-049) for financial support. Partial financial support from the Villum Fonden via 
the DarkSILD project and from the HyMeCav project (FP7PEOPLE-2013-IRSES N612564) is acknowledged.

\section{APPENDIX A: WAVES IN MULTILAYERED BIANISOTROPIC MEDIA}

Let us consider the general case of a monochromatic electromagnetic wave (angular frequency $\omega$ ) propagating in a planar bianisotropic slab. Due to the translation invariance in the plane $(x, y)$ the electric field (similarly magnetic field and inductions) can be written as $\mathbf{E}(\mathbf{r})=\mathbf{E}(z) \exp \left(i k_{0} \mathbf{b} \cdot \mathbf{r}\right)$, where $\mathbf{b}=\mathbf{k}_{t} / k_{0}$ is the normalized tangential wave vector (see Fig. 1). The bianisotropic medium is characterized by dielectric permittivity tensor $\hat{\varepsilon}$, magnetic permeability tensor $\hat{\mu}$, and gyration pseudotensors $\hat{\alpha}$ and $\hat{\beta}[38,39]$ according to the constitutive equations

$$
\mathbf{D}=\hat{\varepsilon}(\omega) \mathbf{E}+\hat{\alpha}(\omega) \mathbf{H}, \quad \mathbf{B}=\hat{\beta}(\omega) \mathbf{E}+\hat{\mu}(\omega) \mathbf{H},
$$

where $\mathbf{E}, \mathbf{H}, \mathbf{D}$, and $\mathbf{B}$ are, respectively, the strengths of the electric and magnetic fields, electric displacement, and magnetic induction. Generally speaking, the layered medium should be local only in the $z$ direction but can possess spatial dispersion in the plane of layers. This means that the material parameters depend on the normalized tangential wave vector b, e.g., as $\varepsilon(\omega, \mathbf{b})$.

Maxwell's equations can be reduced to the four firstorder ordinary differential equations for the tangential field components $\mathbf{W}=\left(\mathbf{H}_{t}, \mathbf{q} \times \mathbf{E}\right)^{T}$, where $T$ denotes transpose operation, $\mathbf{H}_{t}=\hat{I} \mathbf{H}=\left(H_{x}, H_{y}\right)^{T}$, and $\mathbf{q} \times \mathbf{E}=\left(-E_{y}, E_{x}\right)^{T}$. Thus, the system of four differential equations reads

$$
\frac{d \mathbf{W}(z)}{d z}=i k_{0} \hat{M} \mathbf{W}(z)=i k_{0}\left(\begin{array}{ll}
A & B \\
C & D
\end{array}\right) \mathbf{W}(z) .
$$

Here $4 \times 4$ matrix $\hat{M}$ is a block matrix [40], whose $2 \times 2$ blocks $\hat{A}, \hat{B}, \hat{C}$, and $\hat{D}$ in the case of bianisotropic media take the form

$$
\begin{aligned}
& \hat{A}=\mathbf{q}^{\times} \hat{\alpha} \hat{I}+\mathbf{q}^{\times} \hat{\varepsilon} \mathbf{q} \otimes \mathbf{v}_{3}+\left(\mathbf{b}+\mathbf{q}^{\times} \hat{\alpha} \mathbf{q}\right) \otimes \mathbf{v}_{1}, \\
& \hat{B}=-\mathbf{q}^{\times} \hat{\varepsilon} \mathbf{q}^{\times}+\mathbf{q}^{\times} \hat{\varepsilon} \mathbf{q} \otimes \mathbf{q}^{\times} \mathbf{v}_{4}+\left(\mathbf{b}+\mathbf{q}^{\times} \hat{\alpha} \mathbf{q}\right) \otimes \mathbf{q}^{\times} \mathbf{v}_{2}, \\
& \hat{C}=\hat{I} \hat{\mu} \hat{I}+\hat{I} \hat{\mu} \mathbf{q} \otimes \mathbf{v}_{1}+(-\mathbf{a}+\hat{I} \hat{\beta} \mathbf{q}) \otimes \mathbf{v}_{3}, \\
& \hat{D}=-\hat{I} \hat{\beta} \mathbf{q}^{\times}+\hat{I} \hat{\mu} \mathbf{q} \otimes \mathbf{q}^{\times} \mathbf{v}_{2}+(-\mathbf{a}+\hat{I} \hat{\beta} \mathbf{q}) \otimes \mathbf{q}^{\times} \mathbf{v}_{4} .
\end{aligned}
$$

We introduce the antisymmetric tensor $\mathbf{q}^{\times}$dual to the unit vector $\mathbf{q}$ [40]. In the index notation it can be written by means of the antisymmetric Levi-Civita tensor $\varepsilon_{i j k}$ as $\left(\mathbf{q}^{\times}\right)_{i k}=\varepsilon_{i j k} q_{j}$ (the convention of summation over repeated indices from 1 to 3 is assumed). Vectors $\mathbf{a} / b=(\mathbf{b} \times \mathbf{q}) / b, \mathbf{b} / b$, and $\mathbf{q}$ form the Cartesian basis, as shown in Fig. 1. The other quantities in Eq. (A3) are defined as

$$
\begin{aligned}
\mathbf{v}_{1} & =\delta_{q}\left(\beta_{q} \mathbf{q} \hat{\alpha} \hat{I}-\varepsilon_{q} \mathbf{q} \hat{\mu} \hat{I}-\beta_{q} \mathbf{a}\right), \\
\mathbf{v}_{2} & =\delta_{q}\left(\beta_{q} \mathbf{q} \hat{\mathbf{\varepsilon}} \hat{I}-\varepsilon_{q} \mathbf{q} \hat{\beta} \hat{I}-\varepsilon_{q} \mathbf{a}\right), \\
\mathbf{v}_{3} & =\delta_{q}\left(\alpha_{q} \mathbf{q} \hat{\mu} \hat{I}-\mu_{q} \mathbf{q} \hat{\alpha} \hat{I}+\mu_{q} \mathbf{a}\right), \\
\mathbf{v}_{4} & =\delta_{q}\left(\alpha_{q} \mathbf{q} \hat{\beta} \hat{I}-\mu_{q} \mathbf{q} \hat{\varepsilon} I+\alpha_{q} \mathbf{a}\right), \\
\delta_{q} & =\left(\varepsilon_{q} \mu_{q}-\alpha_{q} \beta_{q}\right)^{-1}, \quad \varepsilon_{q}=\mathbf{q} \hat{\varepsilon} \mathbf{q}, \\
\mu_{q} & =\mathbf{q} \hat{\mu} \mathbf{q}, \quad \alpha_{q}=\mathbf{q} \hat{\alpha} \mathbf{q}, \quad \beta_{q}=\mathbf{q} \hat{\beta} \mathbf{q} .
\end{aligned}
$$

The fundamental solution of Eq. (A2) for a homogeneous bianisotropic medium with constant matrix $\hat{M}$ is the matrix (a)

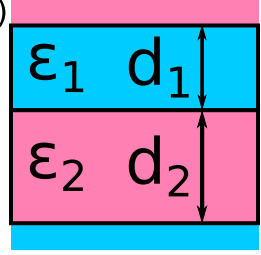

(c)

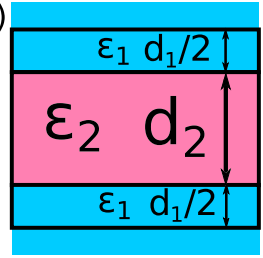

(b)

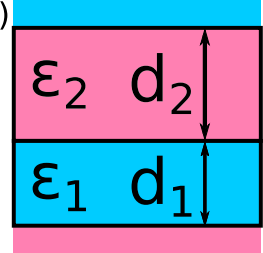

(d)

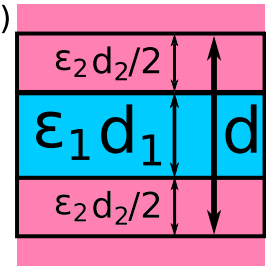

FIG. 7. Schematics of investigated unit cells. (a) Regular and (b) reversed order of layers in bilayer unit cells. (c) Regular and (d) reversed order of three-layer unit cells with the inversion center.

exponential $[40,41]$

$$
\mathbf{W}(z)=\exp \left[i k_{0} z \hat{M}\right] \mathbf{W}(0),
$$

where $\mathbf{W}(0)$ is the initial field at $z=0$. Owing to the continuity of the tangential fields the output field from the first slab $\mathbf{W}$ is the input field for the second one, the initial field for the third slab is produced at the output of the second slab, and so on. Thus, the result of transmission through the stack of $N$ slabs has the form

$$
\mathbf{W}(d)=\prod_{j=1}^{N} \exp \left[i k_{0} d_{j} \hat{M}_{j}\right] \mathbf{W}(0)=\hat{P}(d) \mathbf{W}(0),
$$

where $d=\sum_{j=1}^{N} d_{j}$ is the thickness of the stack and $\hat{P}(d)$ is a spatial evolution operator [42]. It should be noted that the order of multiplication of noncommuting exponentials is important. For instance, the evolution of the field $\mathbf{W}(0)$ passing from top to bottom through the unit cell depicted in Fig. 7(a) is given by the formula

$$
\mathbf{W}(d)=\exp \left[i k_{0} d_{2} \hat{M}_{2}\right] \exp \left[i k_{0} d_{1} \hat{M}_{1}\right] \mathbf{W}(0) .
$$

Here $\hat{M}_{1}$ and $\hat{M}_{2}$ are the $M$ matrices (A2) and (A3) of isotropic media characterized by permittivities $\varepsilon_{1}$ and $\varepsilon_{2}$, respectively. The order of exponentials changes for the unit cell in Fig. 7(b).

\section{APPENDIX B: HOMOGENIZATION USING OEMA}

The idea of the operator effective medium approximation is based on equating the evolution operators of a layered medium and homogenized effective medium. The latter should be characterized by the bianisotropic response in the most general case. Since the evolution operators define the transmission and reflection coefficients [28], the original and homogenized media are not distinguishable. Periodic media allow us just to equate the evolution operators of the unit cell and homogenized effective medium of the same thickness. For a bilayer unit cell we write

$$
\exp \left[i k_{0}\left(d_{1}+d_{2}\right) \hat{M}_{\mathrm{eff}}\right]=\exp \left[i k_{0} d_{2} \hat{M}_{2}\right] \exp \left[i k_{0} d_{1} \hat{M}_{1}\right] .
$$


Using the Baker-Campbell-Hausdorff series [28,31], one obtains the second-order OEMA as

$$
\begin{aligned}
\hat{M}_{\text {eff }}= & \rho \hat{M}_{1}+(1-\rho) \hat{M}_{2} \\
& +\frac{i k_{0} d}{2} \rho(1-\rho)\left[\hat{M}_{2}, \hat{M}_{1}\right] \\
& -\frac{\left(k_{0} d\right)^{2}}{12} \rho(1-\rho)\left(\rho\left[\left[\hat{M}_{2}, \hat{M}_{1}\right], \hat{M}_{1}\right]\right. \\
& \left.+(1-\rho)\left[\left[\hat{M}_{1}, \hat{M}_{2}\right], \hat{M}_{2}\right]\right),
\end{aligned}
$$

where $d=d_{1}+d_{2}$ and $\left[\hat{M}_{1}, \hat{M}_{2}\right]$ is a commutator. In the zeroth-order OEMA we keep only the terms with $\left(k_{0} d\right)^{0}$; that is, the effective material parameters following from the equation $\hat{M}_{\text {eff }}=\rho \hat{M}_{1}+(1-\rho) \hat{M}_{2}$ lead to the Maxwell Garnett approximation (1). With the second-order OEMA the Maxwell Garnett components of the permittivity and permeability tensors are corrected as follows:

$$
\begin{aligned}
\varepsilon_{\|}^{(2)} & =\varepsilon_{\|}^{(0)}+\frac{\left(k_{0} d\right)^{2}}{6} \sigma f(b) \tilde{\varepsilon}_{\|}, \\
\mu_{\|}^{(2)} & =1+\frac{\left(k_{0} d\right)^{2}}{6} \sigma(2 \rho-1), \\
\frac{1}{\varepsilon_{\perp}^{(2)}} & =\frac{1}{\varepsilon_{\perp}^{(0)}}+\frac{\left(k_{0} d\right)^{2}}{6} \sigma\left(\frac{2 \rho-1}{\varepsilon_{r}}-\frac{f(b)}{\tilde{\varepsilon}_{\perp}}\right), \\
\frac{1}{\mu_{\perp}^{(2)}} & =1+\frac{\left(k_{0} d\right)^{2}}{6} \sigma\left(\rho \frac{\varepsilon_{1}}{\varepsilon_{2}}-(1-\rho) \frac{\varepsilon_{2}}{\varepsilon_{1}}\right),
\end{aligned}
$$

where

$$
\begin{aligned}
\tilde{\varepsilon}_{\| \mid} & =\rho \varepsilon_{1}-(1-\rho) \varepsilon_{2}, \quad \tilde{\varepsilon}_{\perp}=\left(\frac{\rho}{\varepsilon_{1}}-\frac{1-\rho}{\varepsilon_{2}}\right)^{-1}, \\
f(b) & =\frac{b^{2}}{\varepsilon_{r}}-1, \quad \varepsilon_{r}=\left(\varepsilon_{1}^{-1}+\varepsilon_{2}^{-1}\right)^{-1}, \\
\sigma & =\rho(1-\rho)\left(\varepsilon_{2}-\varepsilon_{1}\right) .
\end{aligned}
$$

For bilayer-cell multilayers the set of effective parameters should be completed with the gyration pseudotensors (3).

In the case of the three-layer unit cell we also equate the evolution operators as follows [for the case depicted in Fig. 7(d)]:

$$
\begin{aligned}
& \exp \left[i k_{0}\left(d_{1}+d_{2}\right) \hat{M}_{\mathrm{eff}}\right] \\
& \quad=\exp \left[\frac{i k_{0} d_{2}}{2} \hat{M}_{2}\right] \exp \left[i k_{0} d_{1} \hat{M}_{1}\right] \exp \left[\frac{i k_{0} d_{2}}{2} \hat{M}_{2}\right] .
\end{aligned}
$$

Then one can also derive $M_{\text {eff }}$ as a series with respect to $k_{0} d$. As expected, the zeroth-order OEMA will result in the same nonmagnetic nongyrotropic effective medium with parameters (1) as for the bilayer-cell structure. The secondorder OEMA introduces the following corrections to the Maxwell Garnett permittivity and permeability tensors:

$$
\begin{aligned}
\varepsilon_{\|}^{(2)} & =\varepsilon_{\|}^{(0)}-\frac{\left(k_{0} d\right)^{2}}{6} \sigma f(b) \varepsilon_{\|}^{\prime}, \\
\mu_{\|}^{(2)} & =1-\frac{\left(k_{0} d\right)^{2}}{12} \sigma(2-\rho), \\
\frac{1}{\varepsilon_{\perp}^{(2)}} & =\frac{1}{\varepsilon_{\perp}^{(0)}}-\frac{\left(k_{0} d\right)^{2}}{6} \sigma\left(\frac{2-\rho}{2 \varepsilon_{r}}-\frac{f(b)}{\varepsilon_{\perp}^{\prime}}\right), \\
\frac{1}{\mu_{\perp}^{(2)}} & =1+\frac{\left(k_{0} d\right)^{2}}{12} \sigma(2-\rho),
\end{aligned}
$$

where

$$
\varepsilon_{\|}^{\prime}=\frac{\rho}{2} \varepsilon_{1}+(1-\rho) \varepsilon_{2}, \quad \varepsilon_{\perp}^{\prime}=\left(\frac{\rho}{2 \varepsilon_{1}}+\frac{1-\rho}{\varepsilon_{2}}\right)^{-1} .
$$

In contrast to the bilayer-cell structure, the artificial magnetic response of the three-layer-cell effective medium is fairly isotropic because $\left(\mu_{\|}^{(2)} / \mu_{\perp}^{(2)}-1\right) \sim\left(k_{0} d\right)^{4}$.

\section{APPENDIX C: CALCULATION OF SURFACE IMPEDANCES OF TE AND TM EIGENMODES}

Wave impedances of TE- and TM-polarized eigenwaves are defined as

$$
\mathbf{q} \times \mathbf{E}_{t}^{m}=Z_{m} \mathbf{H}_{t}^{m},
$$

where $m$ designates TE and TM polarizations, $\mathbf{E}_{t}$ and $\mathbf{H}_{t}$ are the tangential components of electric and magnetic fields, respectively, at the interface $z=0$, and $Z$ is the wave impedance. Tangential magnetic fields for TE- and TMpolarized eigenwaves in a homogeneous medium can be written as $\mathbf{H}_{t m}=H_{0 m} \exp \left(i k_{0} n_{m} z\right) \mathbf{h}_{m}$, where $m=(\mathrm{TE}, \mathrm{TM})$, $H_{0 m}$ is the amplitude, $n_{m}$ is the refractive index, $\mathbf{h}_{\mathrm{TE}}=\mathbf{b}$, and $\mathbf{h}_{\mathrm{TM}}=\mathbf{a}$. Using the definition of impedance $(\mathrm{C} 1)$, the electric field reads $\mathbf{q} \times \mathbf{E}_{t m}=H_{0 m} \exp \left(i k_{0} n_{m} z\right) Z_{m} \mathbf{h}_{m}$. By substituting these tangential fields into differential equation (A2) we obtain the system of algebraic equations

$$
n_{m}=A_{m}+B_{m} Z_{m}, \quad Z_{m} n_{m}=C_{m}+D_{m} Z_{m},
$$

where $A_{m}=\mathbf{h}_{m} \hat{A} \mathbf{h}_{m}, \quad B_{m}=\mathbf{h}_{m} \hat{B} \mathbf{h}_{m}, \quad C_{m}=\mathbf{h}_{m} \hat{C} \mathbf{h}_{m}$, and $D_{m}=\mathbf{h}_{m} \hat{D} \mathbf{h}_{m}$. The ultimate expression for the surface impedance takes the form

$$
Z_{m}=\frac{D_{m}-A_{m} \pm \sqrt{\left(A_{m}-D_{m}\right)^{2}+4 B_{m} C_{m}}}{2 B_{m}},
$$

where the plus and minus signs correspond to the forward and backward waves, respectively. If TE and TM waves are not the eigenmodes of the media, then generalization of the wave impedance notion is required (e.g., see Ref. [41]).

In the homogeneous medium characterized by material parameter equations (2) and (3), the impedances can be derived as

$$
Z_{\mathrm{TE}}^{ \pm}=\frac{\mu_{\|}}{i \alpha_{\mathrm{TE}}+i \kappa_{\mathrm{TE}}^{ \pm}}, \quad Z_{\mathrm{TM}}^{ \pm}=\frac{i \alpha_{\mathrm{TM}}+i \kappa_{\mathrm{TM}}^{ \pm}}{\varepsilon_{\|}},
$$

where

$$
\begin{aligned}
& \kappa_{\mathrm{TE}}^{ \pm}= \pm \sqrt{\left(\frac{\mu_{\|}}{\mu_{\perp}}\right)\left(b^{2}+\alpha_{\mathrm{TE}}^{2} \frac{\mu_{\perp}}{\mu_{\|}}-\varepsilon_{\|} \mu_{\perp}\right)}, \\
& \kappa_{\mathrm{TM}}^{ \pm}= \pm \sqrt{\left(\frac{\varepsilon_{\|}}{\varepsilon_{\perp}}\right)\left(b^{2}+\alpha_{\mathrm{TM}}^{2} \frac{\varepsilon_{\perp}}{\varepsilon_{\|}}-\varepsilon_{\perp} \mu_{\|}\right)} .
\end{aligned}
$$

The signs are chosen to ensure field localization at the interface.

General equation (C4) can be applied to isotropic dielectric and effective media in the zeroth, first, and second orders of OEMA. In the case of the isotropic dielectric with permittivity $\varepsilon$, the attenuation constant is polarization independent, and Eqs. (C4) and (C5) are reduced to

$$
Z_{\mathrm{TE}}^{ \pm}=\frac{1}{i \kappa^{ \pm}}, \quad Z_{\mathrm{TM}}^{ \pm}=\frac{i \kappa^{ \pm}}{\varepsilon}, \quad \kappa^{ \pm}= \pm \sqrt{b^{2}-\varepsilon} .
$$




\section{APPENDIX D: EVOLUTION OPERATORS AND IMPEDANCES OF UNIT CELLS}

Here we present analytical expressions for the evolution operators of periodic structures with alternating isotropic slabs with permittivities $\varepsilon_{1}$ and $\varepsilon_{2}$ depicted in Fig. 7. The evolution operator for both bilayer and three-layer unit cells takes the same form,

$$
\hat{P}(d)=\left(\begin{array}{cccc}
A_{\mathrm{TE}} & 0 & i B_{\mathrm{TE}} & 0 \\
0 & A_{\mathrm{TM}} & 0 & i B_{\mathrm{TM}} \\
i C_{\mathrm{TE}} & 0 & D_{\mathrm{TE}} & 0 \\
0 & i C_{\mathrm{TM}} & 0 & D_{\mathrm{TM}}
\end{array}\right) .
$$

The TM- (TE-) polarized magnetic (electric) field is oriented along the vector $\mathbf{a}$ and can be described by the $2 \times 2$ matrix as

$$
\hat{P}_{m}(d)=\left(\begin{array}{cc}
A_{m} & i B_{m} \\
i C_{m} & D_{m}
\end{array}\right), \quad m=(\mathrm{TE}, \mathrm{TM}) .
$$

The condition of unimodularity of the matrix $\hat{P}_{m}(d)$ reads

$$
A_{\mathrm{TE}} D_{\mathrm{TE}}+B_{\mathrm{TE}} C_{\mathrm{TE}}=1, \quad A_{\mathrm{TM}} D_{\mathrm{TM}}+B_{\mathrm{TM}} C_{\mathrm{TM}}=1 .
$$

In the case of the three-layer unit cell the following equalities are also valid:

$$
A_{\mathrm{TE}}=D_{\mathrm{TE}}, \quad A_{\mathrm{TM}}=D_{\mathrm{TM}} .
$$

Bloch eigenwaves of periodic multilayers gain phase $K_{B} d$ after passing through a unit cell. It can be found as an eigenvalue of the spatial evolution operator

$$
\hat{P}(d) \mathbf{W}(0)=\exp \left[i K_{B} d\right] \mathbf{W}(0),
$$

where $K_{B}$ is the Bloch wave number and $W(0)$ is the field of a Bloch wave on the very top of a multilayer. Thus, in order to find the surface wave impedance of a Bloch wave on the top of the multilayer one has to solve the eigenvalue and eigenvector problem for a spatial evolution operator. General expressions for the TE- and TM-polarized Bloch eigenvectors of operator $\hat{P}(d)$ [see Eq. (D1)] read

$$
\mathbf{W}_{\mathrm{TE}}(0)=\left(\begin{array}{c}
1 \\
0 \\
Z_{\mathrm{TE}} \\
0
\end{array}\right), \quad \mathbf{W}_{\mathrm{TM}}(0)=\left(\begin{array}{c}
0 \\
1 \\
0 \\
Z_{\mathrm{TM}}
\end{array}\right) .
$$

Wave impedances of the eigenwaves are provided by the equation

$$
Z_{m}^{ \pm}=\frac{C_{m}}{i \frac{D_{m}-A_{m}}{2} \pm i \sqrt{\left(\frac{A_{m}+D_{m}}{2}\right)^{2}-1}} .
$$

In the case of the bilayer unit cell shown in Fig. 7(a) one arrives at the following components exploited in the expressions for impedances:

$$
\begin{aligned}
A_{\mathrm{TE}}= & \cos \left(k_{z 1} d_{1}\right) \cos \left(k_{z 2} d_{2}\right)-\frac{k_{z 2}}{k_{z 1}} \sin \left(k_{z 1} d_{1}\right) \sin \left(k_{z 2} d_{2}\right), \\
C_{\mathrm{TE}}= & k_{0}\left[\sin \left(k_{z 2} d_{2}\right) \cos \left(k_{z 1} d_{1}\right) / k_{z 2}\right. \\
& \left.+\sin \left(k_{z 1} d_{1}\right) \cos \left(k_{z 2} d_{2}\right) / k_{z 1}\right], \\
D_{\mathrm{TE}}= & \cos \left(k_{z 1} d_{1}\right) \cos \left(k_{z 2} d_{2}\right)-\frac{k_{z 1}}{k_{z 2}} \sin \left(k_{z 1} d_{1}\right) \sin \left(k_{z 2} d_{2}\right),
\end{aligned}
$$

$$
\begin{aligned}
A_{\mathrm{TM}}= & \cos \left(k_{z 1} d_{1}\right) \cos \left(k_{z 2} d_{2}\right)-\frac{\varepsilon_{1} k_{z 2}}{\varepsilon_{2} k_{z 1}} \sin \left(k_{z 1} d_{1}\right) \sin \left(k_{z 2} d_{2}\right), \\
C_{\mathrm{TM}}= & \frac{1}{k_{0}}\left[\sin \left(k_{z 2} d_{2}\right) \cos \left(k_{z 1} d_{1}\right) \frac{k_{z 2}}{\varepsilon_{2}}\right. \\
& \left.+\sin \left(k_{z 1} d_{1}\right) \cos \left(k_{z 2} d_{2}\right) \frac{k_{z 1}}{\varepsilon_{1}}\right] \\
D_{\mathrm{TM}}= & \cos \left(k_{z 1} d_{1}\right) \cos \left(k_{z 2} d_{2}\right)-\frac{\varepsilon_{2} k_{z 1}}{\varepsilon_{1} k_{z 2}} \sin \left(k_{z 1} d_{1}\right) \sin \left(k_{z 2} d_{2}\right),
\end{aligned}
$$

where $k_{z j}=k_{0} \sqrt{\varepsilon_{j}-b^{2}}(j=1,2)$ is the longitudinal component of the wave vector in the $j$ th layer. In order to change the order of layers [as in Fig. 7(b)] one just has to interchange $A$ and $D$.

Two quantities, $A$ and $C$, define the simplified Bloch wave impedances for the three-layer unit cell

$$
Z_{m}^{ \pm}= \pm \frac{\tilde{C}_{m}}{i \sqrt{\tilde{A}_{m}^{2}-1}}
$$

Dealing with the configuration in Fig. 7(c), one finds the following expressions:

$$
\begin{gathered}
\tilde{A}_{\mathrm{TE}}=\cos \left(k_{z 1} d_{1}\right) \cos \left(k_{z 2} d_{2}\right)-\frac{k_{z 1}^{2}+k_{z 2}^{2}}{2 k_{z 1} k_{z 2}} \sin \left(k_{z 1} d_{1}\right) \sin \left(k_{z 2} d_{2}\right), \\
\tilde{C}_{\mathrm{TE}}=k_{0}\left[\frac{\sin \left(k_{z 1} d_{1}\right) \cos \left(k_{z 2} d_{2}\right)}{k_{z 1}}+\frac{\sin \left(k_{z 2} d_{2}\right)\left[k_{z 1}^{2}-k_{z 2}^{2}+\left(k_{z 1}^{2}+k_{z 2}^{2}\right) \cos \left(k_{z 1} d_{1}\right)\right]}{2 k_{z 1}^{2} k_{z 2}}\right], \\
\tilde{A}_{\mathrm{TM}}=\cos \left(k_{z 1} d_{1}\right) \cos \left(k_{z 2} d_{2}\right)-\frac{1}{2}\left(\frac{\varepsilon_{2} k_{z 1}}{\varepsilon_{1} k_{z 2}}+\frac{\varepsilon_{1} k_{z 2}}{\varepsilon_{2} k_{z 1}}\right) \sin \left(k_{z 1} d_{1}\right) \sin \left(k_{z 2} d_{2}\right), \\
\tilde{C}_{\mathrm{TM}}=\frac{1}{k_{0}}\left[\frac{k_{z 1}}{\varepsilon_{1}} \sin \left(k_{z 1} d_{1}\right) \cos \left(k_{z 2} d_{2}\right)+\frac{\sin \left(k_{z 2} d_{2}\right)\left[\varepsilon_{1}^{2} k_{z 2}^{2}-\varepsilon_{2}^{2} k_{z 1}^{2}+\cos \left(k_{z 1} d_{1}\right)\left(\varepsilon_{2}^{2} k_{z 1}^{2}+\varepsilon_{1}^{2} k_{z 2}^{2}\right)\right]}{2 \varepsilon_{1}^{2} \varepsilon_{2} k_{z 2}}\right] .
\end{gathered}
$$

For the unit cell in Fig. 7(d) one should interchange indices 1 and 2 in the above equations. 
[1] J. Zhang, L. Zhang, and W. Xu, J. Phys. D 45, 113001 (2012).

[2] M. Specht, J. D. Pedarnig, W. M. Heckl, and T. W. Hänsch, Phys. Rev. Lett. 68, 476 (1992).

[3] A. Sinibaldi, N. Danz, E. Descrovi, P. Munzert, U. Schulz, F. Sonntag, L. Dominici, and F. Michelotti, Sens. Actuat. B 174, 292 (2012).

[4] F. Frascella, S. Ricciardi, P. Rivolo, V. Moi, F. Giorgis, E. Descrovi, F. Michelotti, P. Munzert, N. Danz, L. Napione, M. Alvaro, and F. Bussolino, Sensors 13, 2011 (2013).

[5] M. Liscidini, M. Galli, M. Shi, G. Dacarro, M. Patrini, D. Bajoni, and J. E. Sipe, Opt. Lett. 34, 2318 (2009).

[6] M. Ballarini, F. Frascella, F. Michelotti, G. Digregorio, P. Rivolo, V. Paeder, V. Musi, F. Giorgis, and E. Descrovi, Appl. Phys. Lett. 99, 043302 (2011).

[7] J. Polo and A. Lakhtakia, Laser Photon. Rev. 5, 234 (2011).

[8] D. Maystre, P. Lalanne, J.-J. Greffet, J. Aizpurua, R. Hillenbrand, R. Mc Phedran, R. Quidant, A. Bouhelier, G. Colas Des Francs, J. Grandidier, G. Lerondel, J. Plain, and S. Kostcheev, in Plasmonics: From Basics to Advanced Topics, edited by N. B. S. Enoch, Springer Series in Optical Sciences (Springer, Berlin, 2012), p. 321.

[9] V. Agranovich and D. Mills, Surface Polaritons: Electromagnetic Waves at Surfaces and Interfaces (North-Holland, Amsterdam, 1982).

[10] M. I. D'yakonov, JETP 67, 714 (1988).

[11] R. D. Meade, K. D. Brommer, A. M. Rappe, and J. D. Joannopoulos, Phys. Rev. B 44, 10961 (1991).

[12] E. Descrovi, T. Sfez, L. Dominici, W. Nakagawa, F. Michelotti, F. Giorgis, and H.-P. Herzig, Opt. Express 16, 5453 (2008).

[13] P. Yeh, A. Yariv, and C.-S. Hong, J. Opt. Soc. Am. 67, 423 (1977).

[14] I. V. Lindell, S. A. Tretyakov, K. I. Nikoskinen, and S. Ilvonen, Microwave Opt. Technol. Lett. 31, 129 (2001).

[15] D. R. Smith and D. Schurig, Phys. Rev. Lett. 90, 077405 (2003).

[16] L. Ferrari, C. Wu, D. Lepage, X. Zhang, and Z. Liu, Prog. Quantum Electron. 40, 1 (2015).

[17] X. Zhang and Z. Liu, Nat. Mater. 7, 435 (2008).

[18] D. Lu and Z. Liu, Nat. Commun. 3, 1205 (2012).

[19] T. Repän, A. V. Lavrinenko, and S. V. Zhukovsky, Opt. Express 23, 25350 (2015).

[20] Z. Jacob, I. I. Smolyaninov, and E. E. Narimanov, Appl. Phys. Lett. 100, 181105 (2012).

[21] J. Kim, V. P. Drachev, Z. Jacob, G. V. Naik, A. Boltasseva, E. E. Narimanov, and V. M. Shalaev, Opt. Express 20, 8100 (2012).
[22] O. Kidwai, S. V. Zhukovsky, and J. E. Sipe, Opt. Lett. 36, 2530 (2011).

[23] O. Kidwai, S. V. Zhukovsky, and J. E. Sipe, Phys. Rev. A 85, 053842 (2012).

[24] H. Herzig Sheinfux, I. Kaminer, Y. Plotnik, G. Bartal, and M. Segev, Phys. Rev. Lett. 113, 243901 (2014).

[25] A. Andryieuski, S. V. Zhukovsky, and A. V. Lavrinenko, Nanotechnology 26, 184001 (2015).

[26] S. V. Zhukovsky, A. Andryieuski, O. Takayama, E. Shkondin, R. Malureanu, F. Jensen, and A. V. Lavrinenko, Phys. Rev. Lett. 115, 177402 (2015).

[27] A. A. Orlov, P. M. Voroshilov, P. A. Belov, and Y. S. Kivshar, Phys. Rev. B 84, 045424 (2011).

[28] V. Popov, A. V. Lavrinenko, and A. Novitsky, Phys. Rev. B 94, 085428 (2016).

[29] M. G. Silveirinha, Phys. Rev. B 75, 115104 (2007).

[30] A. V. Chebykin, A. A. Orlov, A. V. Vozianova, S. I. Maslovski, Y. S. Kivshar, and P. A. Belov, Phys. Rev. B 84, 115438 (2011).

[31] A. N. Borzdov, in Advances in Electromagnetics of Complex Media and Metamaterials, edited by S. Zouhdi, A. Sihvola, and M. Arsalane, NATO Science Series, Series II (Kluwer Academic, Dordrecht, 2003), Vol. 89, p. 259.

[32] A. Hartstein, E. Burstein, J. Brion, and R. Wallis, Solid State Commun. 12, 1083 (1973).

[33] A. Hartstein, E. Burstein, J. Brion, and R. Wallis, Surf. Sci. 34, 81 (1973).

[34] Y. Fang, L. Chen, N. Zhu, and J. Zhou, IET Optoelectron. 7, 9 (2013).

[35] J. Martorell, D. W. L. Sprung, and G. V. Morozov, J. Opt. A 8, 630 (2006).

[36] M. Kaliteevski, I. Iorsh, S. Brand, R. A. Abram, J. M. Chamberlain, A. V. Kavokin, and I. A. Shelykh, Phys. Rev. B 76, 165415 (2007).

[37] Z. Sun, X. Zuo, T. Guan, and W. Chen, Opt. Express 22, 4714 (2014).

[38] F. Fedorov, Theory of Gyrotropy (Nauka, Moscow, 1976).

[39] S. Tretyakov, Analytical Modeling in Applied Electromagnetics (Artech House, Norwood, MA, 2003).

[40] L. M. Barkovskii, G. N. Borzdov, and A. V. Lavrinenko, J. Phys. A 20, 1095 (1987).

[41] G. N. Borzdov, J. Math. Phys. 38, 6328 (1997).

[42] L. M. Barkovsky, G. N. Borzdov, and F. I. Fedorov, J. Mod. Opt. 37, 85 (1990). 